\title{
OUTPUT FEEDBACK STABILIZATION OF A ONE-DIMENSIONAL WAVE EQUATION WITH AN ARBITRARY TIME DELAY IN BOUNDARY OBSERVATION*
}

\author{
Bao-Zhu Guo ${ }^{1,2,3}$, Cheng-Zhong $\mathrm{Xu}^{4}$ and Hassan Hammouri ${ }^{4}$
}

\begin{abstract}
The stabilization with time delay in observation or control represents difficult mathematical challenges in the control of distributed parameter systems. It is well-known that the stability of closed-loop system achieved by some stabilizing output feedback laws may be destroyed by whatever small time delay there exists in observation. In this paper, we are concerned with a particularly interesting case: Boundary output feedback stabilization of a one-dimensional wave equation system for which the boundary observation suffers from an arbitrary long time delay. We use the observer and predictor to solve the problem: The state is estimated in the time span where the observation is available; and the state is predicted in the time interval where the observation is not available. It is shown that the estimator/predictor based state feedback law stabilizes the delay system asymptotically or exponentially, respectively, relying on the initial data being non-smooth or smooth. Numerical simulations are presented to illustrate the effect of the stabilizing controller.
\end{abstract}

Mathematics Subject Classification. 35B37, 93B52, 93C05, 93C20, 93D15.

Received August 26, 2009. Revised June 15, 2010.

Published online December 2, 2010.

\section{INTRODUCTION}

In a practical control system, there is often a time delay between the controller to be implemented and the information via the observation of the system. A general case occurs in sampled-data control systems. Time delay may destroy the stability or cause periodic oscillations for a control system [9]. For distributed parameter control systems, the stabilization with time delay in observation and control represents difficult mathematical challenges ([8], p. 69). The first example of a one-dimensional wave equation with boundary feedback in $[4,6]$ illustrates that every small time delay in the known stabilizing boundary output feedback schemes could destabilize the system. This interesting case was particularly mentioned in [8], on p. 69, and

\footnotetext{
Keywords and phrases. Wave equation, time delay, observer, predictor, feedback control, stability.

* This work was supported by the National Natural Science Foundation of China, the National Basic Research Program of China (2011CB808002) and the National Research Foundation of South Africa.

1 Academy of Mathematics and Systems Science, Academia Sinica, Beijing 100190, P.R. China. Bao-Zhu.Guo@wits.ac.za

2 School of Mathematical Sciences, Shanxi University, Taiyuan 030006, P.R. China.

${ }^{3}$ School of Computational and Applied Mathematics, University of the Witwatersrand, Wits 2050, Johannesburg, South Africa.

${ }^{4}$ Université de Lyon, LAGEP, Bâtiment CPE, Université Lyon 1, 43 boulevard du 11 Novembre 1918, 69622 Villeurbanne Cedex, France.
} 
subsequently in $[2,3,5]$. When the time delay appears, re-designing a stabilizing controller becomes thereby necessary. The difficulty to overcome in the time delay problem for elastic systems is that there are infinitely many eigen-modes on the imaginary axis, which is in sharp contrast to parabolic systems. A general result shown in [16] for a class of infinite-dimensional control systems is that if there is a time delay in the output, the stabilization by the PI output feedback becomes defective or the stabilization is not robust to time delay. However this does not mean that there is no stabilizing controller in the presence of time delay.

Recently, inspired by the works of $[7,19]$, we solved successfully the stabilization of one dimensional wave and beam equations with boundary control and non-collocated observation [12,14]. The generalization to multidimensional systems is also available [11]. The idea behind is to use the infinite-dimensional observer to recover the state. In the presence of time delay, we used in [13] the same idea for Euler-Bernoulli beam equation: The state is estimated by the observer in the time span where the observation is available; and the state is predicted in the time interval where the observation is not available. Unfortunately, the convergence is only proved for smooth initial values in [13]. One of main objectives of this paper is to deal with the convergence of the scheme for one-dimensional wave equation when the initial values are not smooth.

The system is described by the one-dimensional wave equation with boundary control and collocated observation with time delay:

$$
\left\{\begin{array}{l}
w_{t t}(x, t)-w_{x x}(x, t)=0,0<x<1, t>0, \\
w(0, t)=0, w_{x}(1, t)=u(t), t \geq 0 \\
w(x, 0)=w_{0}(x), w_{t}(x, 0)=w_{1}(x), 0 \leq x \leq 1, \\
y(t)=w_{t}(1, t-\tau), t \geq \tau
\end{array}\right.
$$

where $u$ is the control (or input), $\left(w_{0}, w_{1}\right)$ is the unknown initial state, $\tau>0$ is a given arbitrary long constant time delay, $y$ is the observation (or output) which suffers delay time $\tau$, that is to say, the information concerning the initial condition is available only after time $\tau$.

It is well-known that if the system (1.1) is free of time delay (i.e., $\tau=0$ ), the proportional output feedback control law $u(t)=-k y(t), k>0$, stabilizes exponentially the system. However, if there is a time delay $\tau>0$, then the closed-loop system (1.1) by the output feedback law $u(t)=-k y(t), k>0$, has at least one eigenvalue with positive real part (see [4]) and so instability occurs. In other words, the proportional output feedback stabilizing control law can not tolerate any time delay. The drawback of this feedback control law makes it almost useless for present elastic system control theory or it is extremely dangerous in applications, because usually there is a time delay from the received information to the implemented control. This problem has been pointed out in [8], on p. 69, and the design of a stabilizing controller for the system (1.1) in presence of time delay is the particularly interesting case raised there.

We proceed as follows. In the next section, Section 2, we show that the open-loop system is well-posed in the sense of Salamon [1]. This seems necessary for the design of the observer. Section 3 is devoted to the design of the observer. In Section 4, we design a stabilizing estimated state feedback control law and show that the closed-loop system is asymptotically stable for non-smooth initial values and exponentially stable for smooth values. Finally, some numerical simulations are presented in Section 5 to illustrate the convergence.

\section{WELL-POSEDNESS OF OPEN-LOOP SYSTEM}

Since the time delay is a dynamic system itself, which represents somehow a memory, we have to specify the initial condition for the memory such that the system (1.1) can be determined. To do this, we introduce a new variable [17]

$$
z(x, t)=w_{t}(1, t-\tau x)
$$


Then the system (1.1) becomes

$$
\left\{\begin{array}{l}
w_{t t}(x, t)-w_{x x}(x, t)=0,0<x<1, t>0 \\
w(0, t)=0, w_{x}(1, t)=u(t), t \geq 0 \\
\tau z_{t}(x, t)+z_{x}(x, t)=0,0<x<1, t>0 \\
z(0, t)=w_{t}(1, t), t \geq 0 \\
w(x, 0)=w_{0}(x), w_{t}(x, 0)=w_{1}(x), z(x, 0)=z_{0}(x), 0 \leq x \leq 1 \\
y(t)=z(1, t), t \geq 0
\end{array}\right.
$$

where $z_{0}$ is the initial value of the new variable $z$.

With state variable $\left(w(\cdot, t), w_{t}(\cdot, t), z(\cdot, t)\right)$, we consider the system $(2.1)$ in the energy state space $H=$ $\mathcal{H} \times L^{2}(0,1)$, where $\mathcal{H}=H_{L}^{1}(0,1) \times L^{2}(0,1)$ and $H_{L}^{1}(0,1)=\left\{f \mid f \in H^{1}(0,1), f(0)=0\right\}$. The norm of $\left(w(\cdot, t), w_{t}(\cdot, t), z(\cdot, t)\right)$ in $H$ is defined by the system energy as follows

$$
E(t)=\frac{1}{2}\left\|\left(w(\cdot, t), w_{t}(\cdot, t), z(\cdot, t)\right)\right\|_{H}^{2}=\frac{1}{2} \int_{0}^{1}\left[w_{x}^{2}(x, t)+w_{t}^{2}(x, t)+z^{2}(x, t)\right] \mathrm{d} x .
$$

The norm of $\left(w(\cdot, t), w_{t}(\cdot, t)\right)$ in $\mathcal{H}$ is defined by

$$
\left\|\left(w(\cdot, t), w_{t}(\cdot, t)\right)\right\|_{\mathcal{H}}^{2}=\int_{0}^{1}\left[w_{x}^{2}(x, t)+w_{t}^{2}(x, t)\right] \mathrm{d} x .
$$

The input space and the output space are the same $U=Y=\mathbb{C}$.

The first question is the well-posedness for the system (2.1) in the sense of Salamon. The question is to know if the mapping from each pair of initial data and control input signal to the pair of state and the output observation signal is continuous.

Theorem 2.1. The system (2.1) is well-posed: For any $\left(w_{0}, w_{1}, z_{0}\right) \in H$ and $u \in L_{\mathrm{loc}}^{2}(0, \infty)$, there exists a unique solution to $(2.1)$ such that $\left(w(\cdot, t), w_{t}(\cdot, t), z(\cdot, t)\right) \in C(0, \infty ; H)$, and for each $T>0$, there exists a constant $C_{T}$ such that

$$
\left\|\left(w(\cdot, T), w_{t}(\cdot, T), z(\cdot, T)\right)\right\|^{2}+\int_{0}^{T} y^{2}(t) \mathrm{d} t \leq C_{T}\left[\left\|\left(w_{0}, w_{1}, z_{0}\right)\right\|^{2}+\int_{0}^{T} u^{2}(t) \mathrm{d} t\right] .
$$

Proof. It is well-known that the following system

$$
\left\{\begin{array}{l}
w_{t t}(x, t)-w_{x x}(x, t)=0,0<x<1, t>0 \\
w(0, t)=0, w_{x}(1, t)=u(t), t \geq 0 \\
y_{w}(t)=w_{t}(1, t), t \geq 0
\end{array}\right.
$$

can be written as a second-order system in $\mathcal{H}[10]$ :

$$
\left\{\begin{array}{l}
w_{t t}(\cdot, t)+A w(\cdot, t)+B u(t)=0 \\
y_{w}(t)=-B^{*} w_{t}(\cdot, t)
\end{array}\right.
$$

where

$$
A f=-f^{\prime \prime}, \forall f \in D(A)=\left\{f \in H^{2}(0,1) \cap H_{L}^{1}(0,1) \mid f^{\prime}(1)=0\right\}, B=-\delta(x-1),
$$

and $\delta(\cdot)$ denotes the Dirac distribution. $A$ has eigen-pairs $\left\{\lambda_{n}=(n-1 / 2 \pi) i, \varphi_{n}=\sin (n-1 / 2) \pi x\right\}_{n \in \mathbb{Z}}$. Since $\left|b_{n}\right|=\left|\left\langle B^{*}, \sin (n-1 / 2) \pi x\right\rangle\right|=1$ for any $n \in \mathbb{Z}$, it follows from Proposition 2 of [10] that $B$ is an admissible input operator [21]. A direct computation shows that the transfer function of (2.3) is

$$
H(s)=\frac{\mathrm{e}^{s}-\mathrm{e}^{-s}}{\mathrm{e}^{s}+\mathrm{e}^{-s}}, \forall \operatorname{Re}(s)>0
$$


which is bounded on some open right half complex plane. Hence the system (2.3) is well-posed in the sense of Salamon [1], that is to say, for any $u \in L_{\mathrm{loc}}^{2}(0, \infty)$ and $\left(w_{0}, w_{1}\right) \in \mathcal{H}$, there exists a unique solution $\left(w(\cdot, t), w_{t}(\cdot, t)\right) \in C(0, \infty ; \mathcal{H})$ to $(2.3)$. Moreover, for any $T>0$, there exists a constant $D_{T}$ such that

$$
\left\|\left(w(\cdot, T), w_{t}(\cdot, T)\right)\right\|_{\mathcal{H}}^{2}+\int_{0}^{T}\left|y_{w}(t)\right|^{2} \mathrm{~d} t \leq D_{T}\left[\left\|\left(w_{0}, w_{1}\right)\right\|_{\mathcal{H}}^{2}+\int_{0}^{T}|u(t)|^{2} \mathrm{~d} t\right] .
$$

This together with the analytic expression of the " $z$ " part solution which is obtained by integrating along the characteristic line

$$
z(x, t)=\left\{\begin{array}{c}
z_{0}\left(x-\frac{t}{\tau}\right), x \geq \frac{t}{\tau} \\
w_{t}(1, t-x \tau), x<\frac{t}{\tau},
\end{array}\right.
$$

gives the required result. Since it involves only simple calculations and we omit the details here.

\section{OBSERVER DESIGN}

The well-posedness for system (2.1) claimed in previous section tells us that the $L_{\text {loc }}^{2}(0, \infty)$ input of the system produces $L_{\text {loc }}^{2}(0, \infty)$ output. This point is particularly important in the design of the observer because in observer, the output of the system becomes the input of the observer.

We go back to system (1.1). For any given $t>\tau$, the output signal $\{y(s) \mid s \in[\tau, t]\}$ is known. We proceed two steps by designing observer and predictor to recover the state of the system (1.1).

Step 1: Construct the observer to estimate the state $\left\{\left(w(x, s), w_{s}(x, s)\right), s \in[0, t-\tau]\right\}$ from the known observation $\{y(s+\tau) \mid s \in[0, t-\tau], t>\tau\}$.

Since the observation $\{y(s+\tau), s \in[0, t-\tau]\}$ has been known and $\left\{\left(w(x, s), w_{s}(x, s)\right), s \in[0, t-\tau]\right\}$ satisfies

$$
\left\{\begin{array}{l}
w_{s s}(x, s)-w_{x x}(x, s)=0,0<x<1,0<s<t-\tau, t>\tau \\
w(0, s)=0,0 \leq s \leq t-\tau, t>\tau \\
w_{x}(1, s)=u(s), 0 \leq s \leq t-\tau, t>\tau \\
w(x, 0)=w_{0}(x), w_{s}(x, 0)=w_{1}(x), 0 \leq x \leq 1
\end{array}\right.
$$

we can construct naturally the observer for the system (3.1) by the principle of "copy of the plant and injection of the output":

$$
\left\{\begin{array}{l}
\widehat{w}_{s s}(x, s)-\widehat{w}_{x x}(x, s)=0,0<x<1,0<s<t-\tau, t>\tau \\
\widehat{w}(0, s)=0,0 \leq s \leq t-\tau \\
\widehat{w}_{x}(1, s)=u(s)-k_{1}\left[\widehat{w}_{s}(1, s)-y(s+\tau)\right], 0 \leq s \leq t-\tau, t>\tau, k_{1}>0 \\
\widehat{w}(x, 0)=\widehat{w}_{0}(x), \widehat{w}_{s}(x, 0)=\widehat{w}_{1}(x), 0 \leq x \leq 1
\end{array}\right.
$$

where $\left(\widehat{w}_{0}, \widehat{w}_{1}\right)$ is an arbitrary initial state of the observer. We first fix the solvability of the observer (3.2).

Proposition 3.1. The system (3.2) is well-posed: For any $t>\tau,\left(w_{0}, w_{1}\right) \in \mathcal{H},\left(\widehat{w}_{0}, \widehat{w}_{1}\right) \in \mathcal{H}$, and $u \in$ $L_{\text {loc }}^{2}(0, \infty)$, there exists a unique solution to $(3.2)$ such that $\left(\widehat{w}(\cdot, s), \widehat{w}_{s}(\cdot, s)\right) \in C(0, t-\tau ; \mathcal{H})$. Moreover, for any $0<T \leq t-\tau$, there exist positive constants $\widehat{C}_{T}, \widetilde{C}_{T}$ such that

$$
\begin{aligned}
\left\|\left(\widehat{w}(\cdot, T), \widehat{w}_{s}(\cdot, T)\right)\right\|_{\mathcal{H}}^{2} & \leq \widehat{C}_{T}\left[\left\|\left(\widehat{w}_{0}, \widehat{w}_{1}\right)\right\|_{\mathcal{H}}^{2}+\int_{\tau}^{T+\tau} y^{2}(s) \mathrm{d} s+\int_{0}^{T} u^{2}(s) \mathrm{d} s\right] \\
& \leq \widetilde{C}_{T}\left[\left\|\left(w_{0}, w_{1}\right)\right\|_{\mathcal{H}}^{2}+\left\|\left(\widehat{w}_{0}, \widehat{w}_{1}\right)\right\|_{\mathcal{H}}^{2}+\int_{0}^{T} u^{2}(s) \mathrm{d} s\right] .
\end{aligned}
$$


Proof. Firstly, notice that (3.2) can be written as

$$
\widehat{w}_{s s}(\cdot, s)+A \widehat{w}(\cdot, s)+k_{1} B B^{*} \widehat{w}_{s}(\cdot, s)+B\left[u(s)+k_{1} y(s+\tau)\right]=0, \forall 0<s<t-\tau, t>\tau,
$$

where $A, B$ are defined in (2.5). By Corollary 1 of [10], (3.3) is well-posed, that is, for any $\left(\widehat{w}_{0}, \widehat{w}_{1}\right) \in \mathcal{H}$, $u(\cdot) \in L_{\text {loc }}^{2}(0, \infty), y(\cdot+\tau) \in L^{2}(0, t-\tau)$ which is assured by Theorem 2.1 , there exists a unique solution to (3.3) such that $\left(\widehat{w}(\cdot, s), \widehat{w}_{s}(\cdot, s)\right) \in C(0, t-\tau ; \mathcal{H})$. Moreover, for any $0<T \leq t-\tau$, there exists a constant $\widehat{C}_{1 T}$ such that

$$
\left\|\left(\widehat{w}(\cdot, T), \widehat{w}_{s}(\cdot, T)\right)\right\|_{\mathcal{H}}^{2} \leq \widehat{C}_{1 T}\left[\left\|\left(\widehat{w}_{0}, \widehat{w}_{1}\right)\right\|_{\mathcal{H}}^{2}+\int_{\tau}^{T+\tau} y^{2}(s) \mathrm{d} s+\int_{0}^{T} u^{2}(s) \mathrm{d} s\right]
$$

This is the first inequality required. The second inequality follows from the first inequality and (2.6) that

$$
\int_{\tau}^{T+\tau}|y(s)|^{2} \mathrm{~d} s \leq D_{T}\left[\left\|\left(w_{0}, w_{1}\right)\right\|_{\mathcal{H}}^{2}+\int_{0}^{T}|u(t)|^{2} \mathrm{~d} t\right]
$$

The proof is complete.

In order for (3.2) to be an observer for (3.1), we have to show its convergence. To do this, let

$$
\varepsilon(x, s)=\widehat{w}(x, s)-w(x, s), 0 \leq s \leq t-\tau .
$$

Then by (3.1), (3.2) and (2.7), $\varepsilon$ satisfies

$$
\left\{\begin{array}{l}
\varepsilon_{s s}(x, s)-\varepsilon_{x x}(x, s)=0,0<x<1,0<s<t-\tau, t>\tau \\
\varepsilon(0, s)=0,0 \leq s \leq t-\tau, t>\tau \\
\varepsilon_{x}(1, s)=-k_{1} \varepsilon_{s}(1, s), 0 \leq s \leq t-\tau, t>\tau \\
\varepsilon(x, 0)=\widehat{w}_{0}(x)-w_{0}(x), \varepsilon_{s}(x, 0)=\widehat{w}_{1}(x)-w_{1}(x), 0 \leq x \leq 1
\end{array}\right.
$$

System (3.6) can be written as:

$$
\frac{\mathrm{d}}{\mathrm{d} s}\left(\begin{array}{c}
\varepsilon(x, s) \\
\varepsilon_{s}(x, s)
\end{array}\right)=\mathcal{A}_{k_{1}}\left(\begin{array}{c}
\varepsilon(x, s) \\
\varepsilon_{s}(x, s)
\end{array}\right)
$$

where the operator $\mathcal{A}_{k_{1}}$ is defined as follows:

$$
\left\{\begin{array}{l}
\mathcal{A}_{k_{1}}(f, g)^{\top}=\left(g, f^{\prime \prime}\right)^{\top} \\
D\left(\mathcal{A}_{k_{1}}\right)=\left\{(f, g)^{\top} \in\left(H^{2}(0,1) \cap H_{L}^{1}(0,1)\right) \times H_{L}^{1}(0,1) \mid f^{\prime}(1)=-k_{1} g(1)\right\} .
\end{array}\right.
$$

It is well-known that $\mathcal{A}_{k_{1}}$ generates an exponential stable $C_{0}$-semigroup on $\mathcal{H}$, which satisfies

$$
\left\|\mathrm{e}^{\mathcal{A}_{k_{1}} t}\right\| \leq M \mathrm{e}^{-\omega t}, \forall t \geq 0
$$

for some positive constants $M, \omega$. Hence, for any $\left(w_{0}, w_{1}\right) \in \mathcal{H},\left(\widehat{w}_{0}, \widehat{w}_{1}\right) \in \mathcal{H}$, there exists a unique solution to (3.6) which satisfies

$$
\left\|\left(\varepsilon(\cdot, s), \varepsilon_{s}(\cdot, s)\right)\right\|_{\mathcal{H}} \leq M \mathrm{e}^{-\omega s}\left\|\left(\widehat{w}_{0}-w_{0}, \widehat{w}_{1}-w_{1}\right)\right\|_{\mathcal{H}}, \forall s \in[0, t-\tau], t>\tau .
$$

Therefore, (3.2) is indeed an observer for (3.1).

Step 2: Predict $\left\{\left(w(x, s), w_{s}(x, s)\right), s \in(t-\tau, t]\right\}$ by $\left\{\left(\widehat{w}(x, s), \widehat{w}_{s}(x, s)\right), s \in[0, t-\tau]\right\}$. 
This is done by solving $(1.1)$ in $[t-\tau, t]$ with estimated initial value $\left(\widehat{w}(x, t-\tau), \widehat{w}_{s}(x, t-\tau)\right)$ obtained from (3.2):

$$
\left\{\begin{array}{l}
\widehat{w}_{s s}^{t}(x, s)-\widehat{w}_{x x}^{t}(x, s)=0,0<x<1, t-\tau<s<t, t>\tau \\
\widehat{w}^{t}(0, s)=0, t-\tau \leq s \leq t, t>\tau \\
\widehat{w}_{x}^{t}(1, s)=u(s), t-\tau \leq s \leq t, t>\tau \\
\widehat{w}^{t}(x, t-\tau)=\widehat{w}(x, t-\tau), \widehat{w}_{s}^{t}(x, t-\tau)=\widehat{w}_{s}(x, t-\tau), 0 \leq x \leq 1, t>\tau
\end{array}\right.
$$

By Theorem 2.1, system (3.11) is well-posed: For any $t>\tau$ and $u \in L^{2}(t-\tau, t)$, there exists a unique solution to (3.11) such that $\left(\widehat{w}^{t}(\cdot, s), \widehat{w}_{t}^{t}(\cdot, s)\right) \in C(t-\tau, t ; \mathcal{H})$. Moreover, for any $s \in[t-\tau, t]$, there exists a constant $\widehat{C}_{t, s}$ such that

$$
\left\|\left(\widehat{w}^{t}(\cdot, s), \widehat{w}_{s}^{t}(\cdot, s)\right)\right\|_{\mathcal{H}}^{2} \leq \widehat{C}_{t, s}\left[\left\|\left(\widehat{w}(\cdot, t-\tau), \widehat{w}_{s}(\cdot, t-\tau)\right)\right\|_{\mathcal{H}}^{2}+\int_{t-\tau}^{t}|u(s)|^{2} \mathrm{~d} s\right] .
$$

This together with Proposition 3.1 leads to the well-posedness of (3.11).

Proposition 3.2. The system (3.11) is well-posed: For any $t>\tau,\left(w_{0}, w_{1}\right) \in \mathcal{H},\left(\widehat{w}_{0}, \widehat{w}_{1}\right) \in \mathcal{H}$, and $u \in$ $L^{2}(t-\tau, t)$, there exists a unique solution to (3.11) such that

$$
\left(\widehat{w}^{t}(\cdot, s), \widehat{w}_{s}^{t}(\cdot, s)\right) \in C([t-\tau, t] ; \mathcal{H})
$$

Moreover, for any $s \in(t-\tau, t]$, there exists a constant $C_{t, s}$ such that

$$
\left\|\left(\widehat{w}^{t}(\cdot, s), \widehat{w}_{s}^{t}(\cdot, s)\right)\right\|_{\mathcal{H}}^{2} \leq C_{t, s}\left[\left\|\left(w_{0}, w_{1}\right)\right\|_{\mathcal{H}}^{2}+\left\|\left(\widehat{w}_{0}, \widehat{w}_{1}\right)\right\|_{\mathcal{H}}^{2}+\int_{0}^{t} u^{2}(s) \mathrm{d} s\right] .
$$

We finally get the estimated state variable:

$$
\widetilde{w}(x, t)=\widehat{w}^{t}(x, t), \widetilde{w}_{t}(x, t)=\widehat{w}_{s}^{t}(x, t), \forall t>\tau .
$$

Theorem 3.1. For any $t>\tau$, we have

$$
\left\|\left(w(\cdot, t)-\widetilde{w}(\cdot, t), w_{t}(\cdot, t)-\widetilde{w}_{t}(\cdot, t)\right)\right\|_{\mathcal{H}} \leq M \mathrm{e}^{-\omega(t-\tau)}\left\|\left(\widehat{w}_{0}-w_{0}, \widehat{w}_{1}-w_{1}\right)\right\|_{\mathcal{H}},
$$

where $\left(\widehat{w}_{0}, \widehat{w}_{1}\right)$ is the initial state of the observer $(3.2),\left(w_{0}, w_{1}\right)$ is the initial state of the original system (1.1), and the positive constants $M, \omega$ are given in (3.10).

Proof. Let

Then $\varepsilon^{t}(x, s)$ satisfies

$$
\varepsilon^{t}(x, s)=\widehat{w}^{t}(x, s)-w(x, s), t-\tau \leq s \leq t .
$$

$$
\left\{\begin{array}{l}
\varepsilon_{s s}^{t}(x, s)-\varepsilon_{x x}^{t}(x, s)=0,0<x<1, t-\tau<s \leq t, t>\tau, \\
\varepsilon^{t}(0, s)=0, t-\tau \leq s \leq t, t>\tau \\
\varepsilon_{x}^{t}(1, s)=0, t-\tau \leq s \leq t, t>\tau, \\
\varepsilon^{t}(x, t-\tau)=\varepsilon(x, t-\tau), \varepsilon_{s}^{t}(x, t-\tau)=\varepsilon_{t}(x, t-\tau), 0 \leq x \leq 1, t>\tau,
\end{array}\right.
$$

which is a conservative system

$$
\left\|\left(\varepsilon^{t}(\cdot, t), \varepsilon_{s}^{t}(\cdot, t)\right)\right\|_{\mathcal{H}}=\left\|\left(\varepsilon(\cdot, t-\tau), \varepsilon_{t}(\cdot, t-\tau)\right)\right\|_{\mathcal{H}} .
$$

This together with (3.10) and (3.12) gives (3.13). 


\section{Stabilization By the EStimated State FeEdBack}

Since $u(t)=-k_{2} w_{t}(1, t)$ stabilizes exponentially the system (1.1), and due to (3.12) and Theorem 3.1, the estimation $\widetilde{w}_{t}(1, t)$ of $w_{t}(1, t)$ has been obtained, we naturally design the observer/predictor based state feedback control law as follows:

$$
u^{*}(t)=\left\{\begin{aligned}
-k_{2} \widetilde{w}_{t}(1, t)=-k_{2} \widehat{w}_{s}^{t}(1, t), & t>\tau, \\
0, & 0 \leq t \leq \tau .
\end{aligned}\right.
$$

By applying this control law the closed-loop system becomes a system of partial differential equations (4.2)-(4.4):

$$
\begin{gathered}
\left\{\begin{array}{l}
w_{t t}(x, t)-w_{x x}(x, t)=0,0<x<1, t>\tau, \\
w(0, t)=0, t>\tau, \\
w_{x}(1, t)=-k_{2} \widehat{w}_{s}^{t}(1, t), t>\tau,
\end{array}\right. \\
\left\{\begin{array}{l}
\widehat{w}_{s s}(x, s)-\widehat{w}_{x x}(x, s)=0,0<x<1,0<s<t-\tau, t>\tau, \\
\widehat{w}(0, s)=0,0 \leq s \leq t-\tau, t>\tau, \\
\widehat{w}_{x}(1, s)=u^{*}(s)-k_{1}\left[\widehat{w}_{s}(1, s)-w_{t}(1, s)\right], 0 \leq s \leq t-\tau, t>\tau, k_{1}>0, \\
\widehat{w}(x, 0)=\widehat{w}_{0}(x), \widehat{w}_{s}(x, 0)=\widehat{w}_{1}(x), 0 \leq x \leq 1
\end{array}\right. \\
\left\{\begin{array}{l}
\widehat{w}_{s s}^{t}(x, s)-\widehat{w}_{x x}^{t}(x, s)=0,0<x<1, t-\tau<s<t, t>\tau, \\
\widehat{w}^{t}(0, s)=0, t-\tau \leq s \leq t, t>\tau, \\
\widehat{w}_{x}^{t}(1, s)=u^{*}(s), t-\tau \leq s \leq t, t>\tau, \\
\widehat{w}^{t}(x, t-\tau)=\widehat{w}(x, t-\tau), \widehat{w}_{s}^{t}(x, t-\tau)=\widehat{w}_{s}(x, t-\tau), 0 \leq x \leq 1, t>\tau .
\end{array}\right.
\end{gathered}
$$

We consider the closed-loop system (4.2)-(4.4) in the state space $X=\mathcal{H}^{3}$. Obviously the system (4.2)-(4.4) is equivalent to the following (4.5)-(4.7) for $t>\tau$ (see also Rem. 4.1 below)

$$
\begin{gathered}
\left\{\begin{array}{l}
w_{t t}(x, t)-w_{x x}(x, t)=0,0<x<1, t>\tau, \\
w(0, t)=0, t>\tau, \\
w_{x}(1, t)=-k_{2} w_{t}(1, t)-k_{2} \varepsilon_{s}^{t}(1, t), t>\tau,
\end{array}\right. \\
\left\{\begin{array}{l}
\varepsilon_{s s}(x, s)-\varepsilon_{x x}(x, s)=0,0<x<1,0<s<t-\tau, t>\tau, \\
\varepsilon(0, s)=0,0 \leq s \leq t-\tau, t>\tau, \\
\varepsilon_{x}(1, s)=-k_{1} \varepsilon_{s}(1, s), 0 \leq s \leq t-\tau, t>\tau, \\
\varepsilon(x, 0)=\widehat{w}_{0}(x)-w_{0}(x), \varepsilon_{s}(x, 0)=\widehat{w}_{1}(x)-w_{1}(x), 0 \leq x \leq 1,
\end{array}\right. \\
\left\{\begin{array}{l}
\varepsilon_{s s}^{t}(x, s)-\varepsilon_{x x}^{t}(x, s)=0,0<x<1, t-\tau<s<t, t>\tau, \\
\varepsilon^{t}(0, s)=0, t-\tau \leq s \leq t, t>\tau, \\
\varepsilon_{x}^{t}(1, s)=0, t-\tau \leq s \leq t, t>\tau, \\
\varepsilon^{t}(x, t-\tau)=\varepsilon(x, t-\tau), \varepsilon_{s}^{t}(x, t-\tau)=\varepsilon_{s}(x, t-\tau), 0 \leq x \leq 1, t>\tau,
\end{array}\right.
\end{gathered}
$$

where $\varepsilon(x, s)$ and $\varepsilon^{t}(x, s)$ are given by (3.5) and (3.14), respectively.

Theorem 4.1. Let $k_{1}>0, k_{2}>0$ and $t>\tau$. Then for any $\left(w_{0}, w_{1}\right) \in \mathcal{H},\left(\widehat{w}_{0}, \widehat{w}_{1}\right) \in \mathcal{H}$, there exists a unique solution to system $(4.5)-(4.7)$ such that $\left(w(\cdot, t), w_{t}(\cdot, t)\right) \in C([\tau, \infty) ; \mathcal{H}),\left(\varepsilon(\cdot, s), \varepsilon_{s}(\cdot, s)\right) \in C([0, t-\tau] ; \mathcal{H})$, $\left(\varepsilon^{t}(\cdot, s), \varepsilon_{s}^{t}(\cdot, s)\right) \in C([t-\tau, t] \times[\tau, \infty) ; \mathcal{H})$. Moreover, for any $t>\tau, s \in[0, t-\tau]$ and $q \in(t-\tau, t]$, there exists a constant $C_{t s q}>0$ such that

$$
\left\|\left(w(\cdot, t), w_{t}(\cdot, t)\right)\right\|_{\mathcal{H}}+\left\|\left(\varepsilon(\cdot, s), \varepsilon_{s}(\cdot, s)\right)\right\|_{\mathcal{H}}+\left\|\left(\varepsilon^{t}(\cdot, q), \varepsilon_{s}^{t}(\cdot, q)\right)\right\|_{\mathcal{H}} \leq C_{t s q}\left[\left\|\left(w_{0}, w_{1}\right)\right\|_{\mathcal{H}}+\left\|\left(\widehat{w}_{0}, \widehat{w}_{1}\right)\right\|_{\mathcal{H}}\right] .
$$

Proof. Firstly, since the operator $\mathcal{A}_{k_{1}}$ defined by (3.8) generates a $C_{0}$-semigroup on $\mathcal{H}$, for any $\left(w_{0}, w_{1}\right) \in \mathcal{H}$, $\left(\widehat{w}_{0}, \widehat{w}_{1}\right) \in \mathcal{H}$, there is a unique solution $\left(\varepsilon(\cdot, s), \varepsilon_{s}(\cdot, s)\right) \in C([0, t-\tau] ; \mathcal{H})$ to $(4.6)$ such that $(3.10)$ holds true. 
Next, for any given time $t>\tau$, since (4.7) is a classical one-dimensional wave equation in $(x, s)$, for any $\left(\varepsilon(\cdot, t-\tau), \varepsilon_{t}(\cdot, t-\tau)\right) \in \mathcal{H}$ that is guaranteed by $(3.10)$, there exists a unique solution to (4.7) such that

$$
\left\|\left(\varepsilon^{t}(\cdot, s), \varepsilon_{s}^{t}(\cdot, s)\right)\right\|_{\mathcal{H}}=\left\|\left(\varepsilon(\cdot, t-\tau), \varepsilon_{t}(\cdot, t-\tau)\right)\right\|_{\mathcal{H}}, \forall s \in[t-\tau, t]
$$

Hence $\left(\varepsilon^{t}(\cdot, s), \varepsilon_{s}^{t}(\cdot, s)\right) \in C([t-\tau, t] \times[t, \infty) ; H)$. Now we consider the well-posedness of $(4.5)$.

For brevity in notation, let us consider the following wave equation:

$$
\left\{\begin{array}{l}
p_{\xi \xi}(x, \xi)-p_{x x}(x, \xi)=0,0<x<1, t-\tau<\xi<\infty \\
p(0, \xi)=p_{x}(1, \xi)=0, t-\tau \leq \xi \leq t \\
p(x, t-\tau)=\varepsilon(x, t-\tau), p_{\xi}(x, t-\tau)=\varepsilon_{s}(x, t-\tau), 0 \leq x \leq 1
\end{array}\right.
$$

Then

Denote by

$$
\varepsilon_{s}^{t}(1, t)=p_{\xi}(1, t)
$$

$$
\widehat{f}(x, s)=\int_{t-\tau}^{\infty} \mathrm{e}^{-s \rho} f(x, \rho) \mathrm{d} \rho
$$

the Laplace transform of the classical (or distribution) function $f(x, \xi)$ where $x$ is regarded as a parameter.

Take Laplace transform to the first equation of (4.9) and take the initial conditions into account, to obtain

$$
\left\{\begin{array}{l}
\frac{\mathrm{d}^{2} \widehat{p}(x, s)}{\mathrm{d} x^{2}}-s^{2} \widehat{p}(x, s)=-s \varepsilon(x, t-\tau)-\varepsilon_{\rho}(x, t-\tau), \\
\widehat{p}(0, s)=\widehat{p}_{x}(1, s)=0
\end{array}\right.
$$

The solution to (4.12) is found to be

$$
\begin{aligned}
\widehat{p}(x, s)= & \frac{\sinh (s x)}{s \cosh (s)} \int_{0}^{1}\left[s \varepsilon(\eta, t-\tau)+\varepsilon_{\rho}(\eta, t-\tau)\right] \cosh (s(1-\eta)) \mathrm{d} \eta \\
& -\frac{1}{s} \int_{0}^{x}\left[s \varepsilon(\eta, t-\tau)+\varepsilon_{\rho}(\eta, t-\tau)\right] \sinh (s(x-\eta)) \mathrm{d} \eta
\end{aligned}
$$

By using $\widehat{p_{\xi}}(x, s)=s \widehat{p}(x, s)-\varepsilon(x, t-\tau)$ and (4.13), a simple computation gives us

$$
\begin{aligned}
\widehat{p_{\xi}}(1, s) & =\int_{0}^{1}\left[s \varepsilon(\eta, t-\tau)+\varepsilon_{\rho}(\eta, t-\tau)\right] \frac{\sinh (s \eta)}{\cosh (s)} \mathrm{d} \eta-\varepsilon(1, t-\tau) \\
& =\int_{0}^{1} \varepsilon_{\rho}(\eta, t-\tau) \frac{\sinh (s \eta)}{\cosh (s)} \mathrm{d} \eta+\frac{1}{\cosh (s)} \int_{0}^{1} \varepsilon(\eta, t-\tau) \mathrm{d} \cosh (s \eta)-\varepsilon(1, t-\tau) \\
& =\int_{0}^{1} \varepsilon_{\rho}(\eta, t-\tau) \frac{\sinh (s \eta)}{\cosh (s)} \mathrm{d} \eta-\int_{0}^{1} \varepsilon_{x}(\eta, t-\tau) \frac{\cosh (s \eta)}{\cosh (s)} \mathrm{d} \eta
\end{aligned}
$$

Using the inverse Laplace transform formulae in [18], on p. 288, we have, for $k=0,1, \ldots$, that

$$
p_{\xi}(1, t)= \begin{cases}-\varepsilon_{x}(4 k+1-\tau, t-\tau)+\varepsilon_{\rho}(4 k+1-\tau, t-\tau) & 4 k \leq \tau<4 k+1 \\ -\varepsilon_{x}(\tau-4 k-1, t-\tau)+\varepsilon_{\rho}(\tau-4 k-1, t-\tau) & 4 k+1 \leq \tau<4 k+2 \\ -\varepsilon_{x}(4 k+3-\tau, t-\tau)+\varepsilon_{\rho}(4 k+3-\tau, t-\tau) & 4 k+2 \leq \tau<4 k+3 \\ -\varepsilon_{x}(\tau-4 k-3, t-\tau)+\varepsilon_{\rho}(\tau-4 k-3, t-\tau) & 4 k+3 \leq \tau<4 k+4\end{cases}
$$


We only treat the case of $4 k \leq \tau<4 k+1$ since other cases can be treated similarly. Let $4 k \leq \tau<4 k+1$. Then $0<4 k+1-\tau \leq 1$. Let

$$
\rho(s)=\int_{0}^{4 k+1-\tau} x \varepsilon_{x}(x, s) \varepsilon_{s}(x, s) \mathrm{d} x, \forall s \in[0, t-\tau] .
$$

Then it is easy to see that $|\rho(s)| \leq \frac{1}{2}\left\|\left(\varepsilon(\cdot, s), \varepsilon_{s}(\cdot, s)\right)\right\|_{\mathcal{H}}^{2}$, and

$$
\dot{\rho}(s)=\frac{4 k+1-\tau}{2}\left[\varepsilon_{s}^{2}(4 k+1-\tau, s)+\varepsilon_{x}^{2}(4 k+1-\tau, s)\right]-\frac{1}{2} \int_{0}^{4 k+1-\tau}\left[\varepsilon_{x}^{2}(x, s)+\varepsilon_{s}^{2}(x, s)\right] \mathrm{d} x .
$$

Integrate above over $[0, T]$ for any $T>0$ to give

$$
\begin{aligned}
& \frac{4 k+1-\tau}{2} \int_{0}^{T}\left[\varepsilon_{s}^{2}(4 k+1-\tau, t)+\varepsilon_{x}^{2}(4 k+1-\tau, t)\right] \mathrm{d} t=\rho(T)-\rho(0) \\
& +\frac{1}{2} \int_{0}^{T} \int_{0}^{4 k+1-\tau}\left[\varepsilon_{x}^{2}(x, t)+\varepsilon_{s}^{2}(x, t)\right] \mathrm{d} x \mathrm{~d} t, \quad \forall T>0 .
\end{aligned}
$$

Using (4.16) and (3.10), we can easily get the following inequality

$$
\begin{aligned}
\int_{0}^{\infty}\left[\varepsilon_{x}^{2}(4 k+1-\tau, t)+\varepsilon_{s}^{2}(4 k+1-\tau, t)\right] \mathrm{d} t & \leq \frac{2 \omega+M^{2}}{(4 k+1-\tau) \omega}\left\|\left(\varepsilon(\cdot, 0), \varepsilon_{s}(\cdot, 0)\right)\right\|_{\mathcal{H}}^{2} \\
& =\frac{2 \omega+M^{2}}{(4 k+1-\tau) \omega}\left\|\left(\widehat{w}_{0}-w_{0}, \widehat{w}_{1}-w_{1}\right)\right\|_{\mathcal{H}}^{2} .
\end{aligned}
$$

This together with (4.10) and (4.15) shows that

$$
\int_{t}^{\infty} \varepsilon_{s}^{t^{2}}(1, \rho) \mathrm{d} \rho \leq \frac{2\left(2 \omega+M^{2}\right)}{(4 k+1-\tau) \omega}\left\|\left(\widehat{w}_{0}-w_{0}, \widehat{w}_{1}-w_{1}\right)\right\|_{\mathcal{H}}^{2}, \forall t>\tau .
$$

Now, since (4.5) can be written as

$$
w_{t t}(\cdot, t)+A w(\cdot, t)+k_{2} B B^{*} w_{t}(\cdot, t)-k_{2} B \varepsilon_{s}^{t}(1, t)=0 \text { for all } t>\tau,
$$

where $A, B$ are defined in (2.4). Similarly to (3.3), (4.19) is well-posed: For any $t>\tau$, there exists a unique solution to (4.19) in $\mathcal{H}$, and there exist constants $C_{t}^{\prime}>0, C_{t}^{\prime \prime}>0$ such that

$$
\begin{aligned}
\left\|\left(w(\cdot, t), w_{t}(\cdot, t)\right)\right\|_{\mathcal{H}}^{2} & \leq C_{t}^{\prime}\left[\left\|\left(w(\cdot, \tau), w_{t}(\cdot, \tau)\right)\right\|_{\mathcal{H}}^{2}+\int_{\tau}^{t} \varepsilon_{s}^{t^{2}}(1, t) \mathrm{d} t\right] \\
& =C_{t}^{\prime}\left[\left\|\left(w_{0}, w_{1}\right)\right\|_{\mathcal{H}}^{2}+\int_{\tau}^{t} \varepsilon_{s}^{t^{2}}(1, t) \mathrm{d} t\right] .
\end{aligned}
$$

This together with (4.8), (4.18) and (3.10) gives the required result.

Remark 4.1. Rigorously, the equivalence of (4.2)-(4.4) and (4.5)-(4.7) requires the control $u^{*}(t)=-k_{2} w_{t}(1, t)-$ $k_{2} \varepsilon_{s}^{t}(1, t), t>\tau$ defined by $(4.1)$ belongs to $L_{\text {loc }}^{2}(\tau, \infty)$. This can be simply proved by defining

$$
G(t)=\frac{1}{2} \int_{0}^{1}\left[w_{t}^{2}(x, t)+w_{x}^{2}(x, t)\right] \mathrm{d} x .
$$


Then we can consider only for the classical solution of (4.5). The reason is the same as in the proof of Lemma A.1 in Appendix,

$$
\dot{G}(t)=-k_{2} w_{t}^{2}(1, t)-k_{2} w_{t}(1, t) \varepsilon_{s}^{t}(1, t) .
$$

Integrate above over $[\tau, T]$ for any $T>\tau$ to give

$$
k_{2} \int_{\tau}^{T} w_{t}^{2}(1, t) \mathrm{d} t=-k_{2} \int_{\tau}^{T} w_{t}(1, t) \varepsilon_{s}^{t}(1, t) \mathrm{d} t-G(T)+G(\tau),
$$

and hence

$$
\frac{k_{2}}{2} \int_{\tau}^{T} w_{t}^{2}(1, t) \mathrm{d} t \leq \frac{k_{2}}{2} \int_{\tau}^{T} \varepsilon_{s}^{t^{2}}(1, t) \mathrm{d} t-G(T)+G(\tau) .
$$

This together with $(4.18)$ and $(4.20)$ shows that $u^{*}(t) \in L_{\mathrm{loc}}^{2}(0, \infty)$.

Finally, we are in a position to show the convergence of the system (4.5).

Theorem 4.2. Let $k_{1}>0$ and let $k_{2}>0$. Then for any initial conditions $\left(w_{0}, w_{1}\right) \in \mathcal{H}$ and $\left(\widehat{w}_{0}, \widehat{w}_{1}\right) \in \mathcal{H}$, the solution of (4.5) satisfies

$$
\lim _{t \rightarrow \infty}\left\|\left(w(\cdot, t), w_{t}(\cdot, t)\right)\right\|_{\mathcal{H}}=0
$$

Furthermore, if $\left(w_{0}, w_{1}\right) \in \mathcal{H}$ and $\left(\widehat{w}_{0}, \widehat{w}_{1}\right) \in \mathcal{H}$ satisfy

$$
\left(w_{0}-\widehat{w}_{0}, w_{1}-\widehat{w}_{1}\right) \in D\left(\mathcal{A}_{k_{1}}\right),
$$

where $\mathcal{A}_{k_{1}}$ is defined by (3.8), then there are some positive constants $M_{2}>0$ and $\omega_{2}>0$ such that the solution of (4.5) satisfies

$$
\left\|\left(w(\cdot, t), w_{t}(\cdot, t)\right)\right\|_{\mathcal{H}} \leq M_{2} \mathrm{e}^{-\omega_{2}(t-\tau)}\left\|\mathcal{A}_{k_{1}}\left(w_{0}-\widehat{w}_{0}, w_{1}-\widehat{w}_{1}\right)\right\|_{\mathcal{H}}, \forall t>\tau
$$

Proof. The first part follows directly from (i) of Proposition A.1 in Appendix. As in the proof of Theorem 4.1, we only consider the case of $4 k \leq \tau<4 k+1$. If $\left(\varepsilon(\cdot, 0), \varepsilon_{s}(\cdot, 0)\right)^{\top} \in D\left(\mathcal{A}_{k_{1}}\right)$, where $\mathcal{A}_{k_{1}}$ is defined by (3.8), then by (3.9)

that is,

$$
\left\|\mathcal{A}_{k_{1}} \mathrm{e}^{\mathcal{A}_{k_{1}} s}\left(\varepsilon(\cdot, 0), \varepsilon_{s}(\cdot, 0)\right)^{\top}\right\|_{\mathcal{H}} \leq M \mathrm{e}^{-\omega s}\left\|\mathcal{A}_{k_{1}}\left(\varepsilon(\cdot, 0), \varepsilon_{s}(\cdot, 0)\right)^{\top}\right\|_{\mathcal{H}}, \forall s \geq 0
$$

$$
\int_{0}^{1}\left[\varepsilon_{x x}^{2}(x, s)+\varepsilon_{x s}^{2}(x, s)\right] \mathrm{d} x \leq M \mathrm{e}^{-\omega s} \int_{0}^{1}\left[\varepsilon_{x x}^{2}(x, 0)+\varepsilon_{x s}^{2}(x, 0)\right] \mathrm{d} x, \forall s \geq 0 .
$$

Since $\varepsilon_{s}(0, s)=0$, it is easy to prove the following inequalities:

$$
\left\{\begin{aligned}
\varepsilon_{s}^{2}(4 k+1-\tau, s) & \leq \int_{0}^{4 k+1-\tau} \varepsilon_{x s}^{2}(x, s) \mathrm{d} x, \quad k_{1}^{-2} \varepsilon_{x}^{2}(1, s)=\varepsilon_{s}^{2}(1, s) \leq \int_{0}^{1} \varepsilon_{x s}^{2}(x, s) \mathrm{d} x, \\
\varepsilon_{x}^{2}(4 k+1-\tau, s) & =\left|\varepsilon_{x}(1, s)+\int_{1}^{4 k+1-\tau} \varepsilon_{x x}(x, s) \mathrm{d} x\right|^{2} \leq 2 \int_{0}^{1}\left[\varepsilon_{x s}^{2}(x, s)+\varepsilon_{x x}^{2}(x, s)\right] \mathrm{d} x .
\end{aligned}\right.
$$

Set now $f(s)=\varepsilon_{s}(1, s, s)$. By (4.10), (4.15), (4.22), (4.23), (ii) of Proposition A.1 in Appendix, and the definition of $D\left(\mathcal{A}_{k_{1}}\right)$, we prove the second part of the required result. 

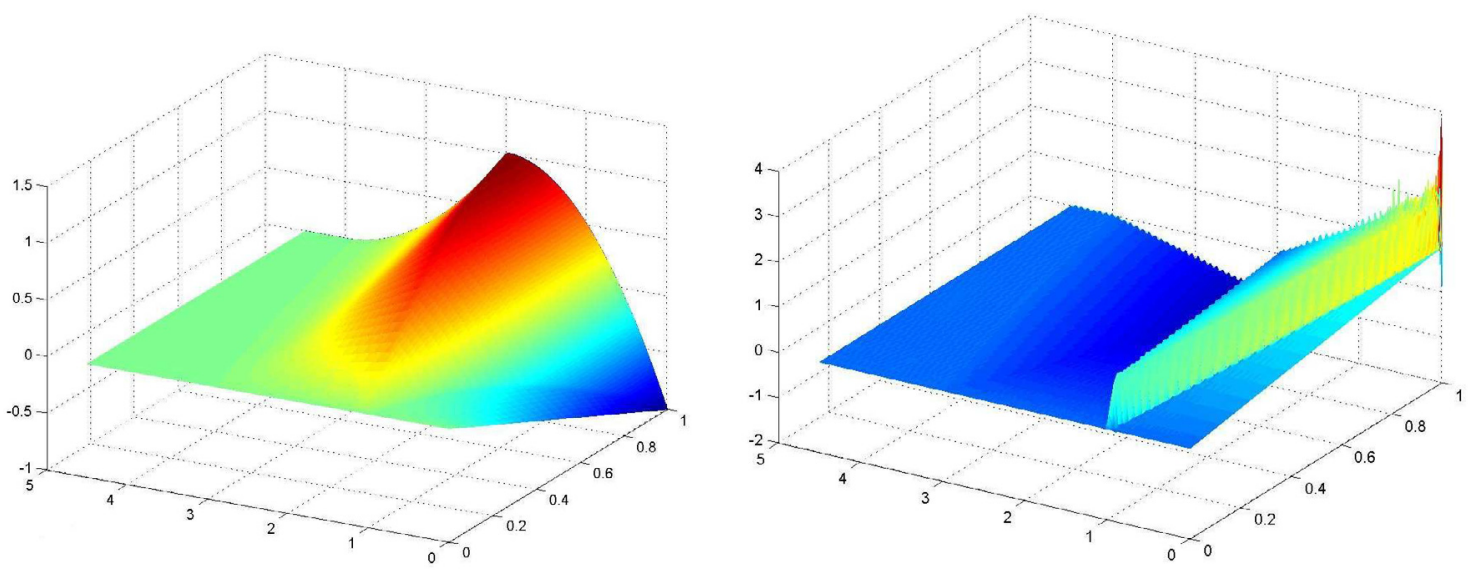

Figure 1. Displacement and velocity with time delay $\tau=0.5$.

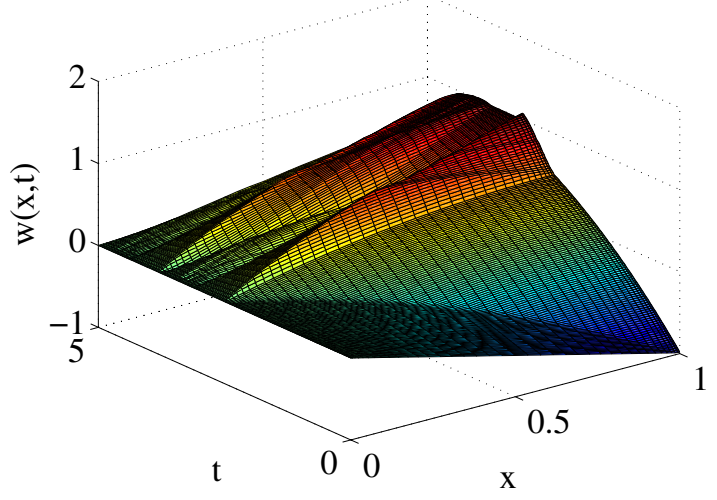

Figure 2. Displacement with time delay $\tau=0.5$ disturbed by $\theta=0.1$.

\section{Simulation Results}

In this section, we give some numerical simulation results for the closed loop systems (4.5)-(4.7). We use the backward Euler method in the time domain and the Chebyshev spectral method in space domain. The numerical results are produced by Matlab program [20].

The basic idea of the numerical scheme is as follows. Firstly, the second order equations in time are transformed into a system of two first order equations. Secondly, the boundary conditions are implemented in an explicit way. In the numerical scheme, the space grid size $N=40$ and $\mathrm{d} t=10^{-4}$ time step are used. The parameters are taken to be $\tau=0.5, k_{1}=k_{2}=1$. The initial conditions are

$$
w_{0}(x)=-x, w_{1}(x)=\frac{x}{k_{2}}, \widehat{w}_{0}(x)=\widehat{w}_{1}(x)=0, \forall x \in[0,1] .
$$

The time span is $[0,4.5]$. It is seen from Figure 1 that both the string displacement and the velocity are almost at rest after $t=4$.

Finally, we point out that our scheme is also not robust to small time delay as the general output PI feedbacks in [16]. In Figure 2, we use grid size $N=40$; time step $\mathrm{d} t=10^{-4}$; parameters $\tau=1.0, k_{1}=k_{2}=1$; and the 
same initial conditions (5.24) to get the displacement where the time delay is disturbed by $\theta=0.1$. It is seen that the displacement is not convergent anymore. This phenomena coincides with [13] for beam equation. The big difference with [13] is that we are able to show the convergence for non-smooth initial values. The scheme is certainly applicable to multi-dimensional PDEs and we believe that the same convergence is also true in that case.

\section{A. Appendix: The solution decay Rate of the nonhomogeneous Wave equation}

Consider the boundary nonhomogeneous wave equation

$$
\left\{\begin{array}{l}
w_{s s}(x, s)-w_{x x}(x, s)=0,0<x<1, s>t \geq 0 \\
w(0, s)=0, s \geq t \\
w_{x}(1, s)=-k w_{s}(1, s)+f(s), s \geq t, k>0
\end{array}\right.
$$

in the space $\mathcal{H}=H_{L}^{1}(0,1) \times L^{2}(0,1), H_{L}^{1}(0,1)=\left\{f \mid f \in H^{1}(0,1), f(0)=0\right\}$.

Proposition A.1. (i) Suppose $f \in L^{2}(t, \infty)$. Then for any initial value $w(\cdot, t), w_{s}(\cdot, t) \in \mathcal{H}$, the solution of (A.25) decays asymptotically to 0 as s goes to infinity:

$$
\lim _{s \rightarrow \infty}\left\|\left(w(\cdot, s), w_{s}(\cdot, s)\right)\right\|_{\mathcal{H}}=0
$$

(ii) Suppose $\mathrm{e}^{\ell s} f(s) \in L^{2}(t, \infty), t \geq 0$ for some $\ell>0$. Then the solution of (A.25) decays exponentially to 0 in $\mathcal{H}$ as follows

$$
\left\|\left(w(\cdot, s), w_{s}(\cdot, s)\right)\right\|_{\mathcal{H}} \leq C_{\mathrm{t}} \mathrm{e}^{-\alpha s}\left[\left\|\left(w(\cdot, t), w_{s}(\cdot, t)\right)\right\|_{\mathcal{H}}+\left\|\mathrm{e}^{\alpha \cdot} f(\cdot)\right\|_{L^{2}(t, \infty)}\right], \forall s \geq t
$$

for some constants $C_{t}>0, \alpha>0$ independent of $s$.

Proof. (i) As (4.19), (A.25) can be written as

$$
w_{s s}(\cdot, s)+A w(\cdot, s)+k B B^{*} w_{s}(\cdot, s)+B f(s)=0 \text { for all } s>t,
$$

where $A, B$ are defined in (2.4). Write (A.26) further as

$$
\frac{\mathrm{d}}{\mathrm{d} s}\left(\begin{array}{c}
w(\cdot, s) \\
w_{s}(\cdot, s)
\end{array}\right)=\mathcal{A}_{k}\left(\begin{array}{c}
w(\cdot, s) \\
w_{s}(\cdot, s)
\end{array}\right)+\mathcal{B}\left(\begin{array}{c}
0 \\
f(s)
\end{array}\right)
$$

where

$$
\mathcal{A}_{k}=\left(\begin{array}{cc}
0 & I \\
-A & -k B B^{*}
\end{array}\right), \mathcal{B}=\left(\begin{array}{c}
0 \\
B
\end{array}\right)
$$

By Proposition 4.2 .10 of $[21]$, on p. 120 , for any $\left(w(\cdot, t), w_{s}(\cdot, t)\right) \in D\left(\mathcal{A}_{k}\right), f \in H_{\mathrm{loc}}^{1}(t, \infty), f(t)=0,(\mathrm{~A} .25)$ admits a unique classical solution.

For any $\left(w(\cdot, t), w_{s}(\cdot, t)\right) \in \mathcal{H}, f \in L^{2}(t, \infty)$ and given $\epsilon>0$, find the $\left(\tilde{w}(\cdot, t), \tilde{w}_{s}(\cdot, t)\right) \in D\left(\tilde{\mathcal{A}}_{k}\right), \tilde{f} \in$ $H_{\text {loc }}^{1}(t, \infty), \tilde{f}(t)=0$ such that

$$
\left\|\left(\tilde{w}(\cdot, t), \tilde{w}_{s}(\cdot, t)\right)-\left(w(\cdot, t), w_{s}(\cdot, t)\right)\right\|_{\mathcal{H}} \leq \epsilon,\|\tilde{f}-f\|_{L^{2}(t, \infty)} \leq \epsilon .
$$

By the well-known facts on (A.26) mentioned in previous sections, $\mathcal{A}_{k}$ and $\mathcal{B}$ satisfies assumptions (H.1)-(H.4) of Theorem 7.4.1.1 of [15], on p. 653. By (7.4.1.3) of Theorem 7.4.1.1 of [15], on p. 654, with $\epsilon=0$, we have

$$
\begin{array}{r}
\left\|\left(\tilde{w}(\cdot, s), \tilde{w}_{s}(\cdot, s)\right)-\left(w(\cdot, s), w_{s}(\cdot, s)\right)\right\|_{\mathcal{H}} \leq C_{t}\left[\left\|\left(\tilde{w}(\cdot, t), \tilde{w}_{s}(\cdot, t)\right)-\left(w(\cdot, t), w_{s}(\cdot, t)\right)\right\|_{\mathcal{H}}\right. \\
\left.+\|\tilde{f}-f\|_{L^{2}(t, \infty)}\right] \leq 2 C_{t} \epsilon, \forall s \geq t
\end{array}
$$


where $C_{t}$ is a constant independent of $\epsilon$ and $\left(\tilde{w}(\cdot, s), \tilde{w}_{s}(\cdot, s)\right)$ is the solution of (A.25) with $f$ replaced by $\tilde{f}$. Therefore, in order to prove (i), we need only consider the classical solution of (A.25).

Now for small $\delta>0$, define the Lyapunov function

$$
F(s)=\frac{1}{2} \int_{0}^{1}\left[w_{s}^{2}(x, s)+w_{x}^{2}(x, s)\right] \mathrm{d} x+\delta \int_{0}^{1} x w_{x}(x, s) w_{s}(x, s) \mathrm{d} x .
$$

Then we have

$$
\begin{aligned}
\dot{F}(s) & =-k w_{s}^{2}(1, s)+f(s) w_{s}(1, s)+\frac{\delta}{2} w_{s}^{2}(1, s)+\frac{\delta}{2} w_{x}^{2}(1, s)-\frac{\delta}{2} \int_{0}^{1}\left[w_{s}^{2}(x, s)+w_{x}^{2}(x, s)\right] \mathrm{d} x \\
& \leq-\delta_{0} F(s)+C_{0} f^{2}(s)
\end{aligned}
$$

for some positive constant $\delta_{0}, C_{0}$ as long as $\delta$ small enough. Hence

$$
F(s) \leq \mathrm{e}^{-\delta_{0}(s-t)} F(t)+C_{0} \int_{t}^{s} \mathrm{e}^{-\delta_{0}(s-\tau)} f^{2}(\tau) \mathrm{d} \tau .
$$

Since $f(s) \in L^{2}(t, \infty)$, for any given $\varepsilon>0$, take $s$ sufficiently large so that $s-t>s / 2$ and $C_{0} \int_{s / 2}^{s} f^{2}(\tau) \mathrm{d} \tau<\varepsilon$. Then

$$
\begin{aligned}
F(s) & \leq \mathrm{e}^{-\delta_{0}(s-t)} F(t)+C_{0} \int_{t}^{s} \mathrm{e}^{-\delta_{0}(s-\tau)} f^{2}(\tau) \mathrm{d} \tau \leq \mathrm{e}^{-\delta_{0}(s-t)} F(t)+C_{0} \int_{t}^{s / 2} \mathrm{e}^{-\delta_{0}(s-\tau)} f^{2}(\tau) \mathrm{d} \tau+\varepsilon \\
& \leq \mathrm{e}^{-\delta_{0}(s-t)} F(t)+C_{0} \mathrm{e}^{-\delta s / 2} \int_{t}^{\infty} f^{2}(\tau) \mathrm{d} \tau+\varepsilon
\end{aligned}
$$

Therefore

$$
\limsup _{s \rightarrow \infty} F(s) \leq \varepsilon
$$

Since $\varepsilon$ is arbitrary chosen, (i) is proved.

Next, we prove (ii). It is well-known that $\mathcal{A}_{k}$ generates an exponential stable $C_{0}$-semigroup:

$$
\left\|\mathrm{e}^{\mathcal{A}_{k} s}\right\| \leq M_{1} \mathrm{e}^{-\omega_{1} s}, \forall s \geq 0
$$

for some $M_{1}>0, \omega_{1}>0$. Suppose $0<\alpha<\min \left\{\ell, \omega_{1}\right\}$ and let

$$
Y(s)=\mathrm{e}^{\alpha s}\left(w(\cdot, s), w_{s}(\cdot, s)\right)^{\top}
$$

Then

$$
\frac{\mathrm{d}}{\mathrm{d} s} Y(s)=\left(\mathcal{A}_{k}+\alpha\right) Y(s)+\mathcal{B}\left(0, \mathrm{e}^{\alpha s} f(s)\right)^{\top} .
$$

Again, $\mathcal{A}_{k}+\alpha$ and $\mathcal{B}$ satisfies assumptions (H.1)-(H.4) of Theorem 7.4.1.1 of [15], on p. 653. Apply (7.4.1.3) of Theorem 7.4.1.1 of [15], on p. 654, with $\epsilon=0$, to obtain

$$
\|Y(s)\|_{\mathcal{H}} \leq C_{t}\left[\left\|\left(w(\cdot, t), w_{s}(\cdot, t)\right)\right\|_{\mathcal{H}}+\left\|\mathrm{e}^{\alpha \cdot f}\right\|_{L^{2}(t, \infty)}\right], \forall s \geq t,
$$

for some $C_{t}>0$. Therefore,

$$
\left\|\left(w(\cdot, s), w_{s}(\cdot, s)\right)\right\|_{\mathcal{H}} \leq C_{t} \mathrm{e}^{-\alpha s}\left[\left\|\left(w(\cdot, t), w_{s}(\cdot, t)\right)\right\|_{\mathcal{H}}+\left\|\mathrm{e}^{\alpha \cdot} f\right\|_{L^{2}(t, \infty)}\right], \forall s \geq t
$$

This is the required result. 
Remark A.1. It should be pointed out that (7.4.1.3) of Theorem 7.4.1.1 of [15], on p. 654, and (7.6.2.8) of Theorem 7.6.2.2 of [15], on p. 665, are valid only for $\epsilon=0$. When $\epsilon>0$, both results are wrong. An counter-example can be constructed as

$$
\dot{x}(t)=-x(t)+u(t), x(0)=1 .
$$

Take $u(t)=\frac{t}{(1+t)^{2}} \in L^{2}(0, \infty)$. Then

$$
x(t)=\mathrm{e}^{-t}+\int_{0}^{\mathrm{t}} \mathrm{e}^{-(t-s)} u(s) \mathrm{d} s=\mathrm{e}^{-t}+[L u](t)=\frac{1}{1+t} .
$$

This shows that (7.4.1.3) of Theorem 7.4.1.1 of [15], on p. 654 , is wrong with $\epsilon>0$.

\section{REFERENCES}

[1] R.F. Curtain, The Salamon-Weiss class of well-posed infinite dimensional linear systems: a survey. IMA J. Math. Control Inform. 14 (1997) 207-223.

[2] R. Datko, Two questions concerning the boundary control of certain elastic systems. J. Diff. Equ. 92 (1991) $27-44$.

[3] R. Datko, Is boundary control a realistic approach to the stabilization of vibrating elastic systems?, in Evolution Equations, Baton Rouge (1992), Lecture Notes in Pure and Appl. Math. 168, Dekker, New York (1995) 133-140.

[4] R. Datko, Two examples of ill-posedness with respect to time delays revisited. IEEE Trans. Automat. Control 42 (1997) $511-515$.

[5] R. Datko and Y.C. You, Some second-order vibrating systems cannot tolerate small time delays in their damping. J. Optim. Theory Appl. 70 (1991) 521-537.

[6] R. Datko, J. Lagnese and M.P. Polis, An example on the effect of time delays in boundary feedback stabilization of wave equations. SIAM J. Control Optim. 24 (1986) 152-156.

[7] A.J. Deguenon, G. Sallet and C.Z. Xu, A Kalman observer for infinite-dimensional skew-symmetric systems with application to an elastic beam, Proc. of the Second International Symposium on Communications, Control and Signal Processing, Marrakech, Morocco (2006).

[8] W.H. Fleming Ed., Future Directions in Control Theory. SIAM, Philadelphia (1988).

[9] I. Gumowski and C. Mira, Optimization in Control Theory and Practice. Cambridge University Press, Cambridge (1968).

[10] B.Z. Guo and Y.H. Luo, Controllability and stability of a second order hyperbolic system with collocated sensor/actuator. Syst. Control Lett. 46 (2002) 45-65.

[11] B.Z. Guo and Z.C. Shao, Stabilization of an abstract second order system with application to wave equations under noncollocated control and observations. Syst. Control Lett. 58 (2009) 334-341.

[12] B.Z. Guo and C.Z. Xu, The stabilization of a one-dimensional wave equation by boundary feedback with non-collocated observation. IEEE Trans. Automat. Contr. 52 (2007) 371-377.

[13] B.Z. Guo and K.Y. Yang, Dynamic stabilization of an Euler-Bernoulli beam equation with time delay in boundary observation. Automatica 45 (2009) 1468-1475.

[14] B.Z. Guo, J.M. Wang and K.Y. Yang, Dynamic stabilization of an Euler-Bernoulli beam under boundary control and noncollocated observation. Syst. Control Lett. 57 (2008) 740-749.

[15] I. Lasiecka and R. Triggiani, Control Theory for Partial Differential Equations: Continuous and Approxiamation Theories II: Abstract Hyperbolic-Like Systems over a Finite Time Horizon. Cambridge University Press, Cambridge (2000).

[16] H. Logemann, R. Rebarber and G. Weiss, Conditions for robustness and nonrobustness of the stability of feedback systems with respect to small delays in the feedback loop. SIAM J. Control Optim. 34 (1996) 572-600.

[17] S. Nicaise and C. Pignotti, Stability and instability results of the wave equation with a delay term in the boundary or internal feedbacks. SIAM J. Control Optim. 45 (2006) 1561-1585.

[18] F. Oberhettinger and L. Badii, Tables of Laplace Transforms. Springer-Verlag, Berlin (1973).

[19] A. Smyshlyaev and M. Krstic, Backstepping observers for a class of parabolic PDEs. Syst. Control Lett. 54 (2005) $613-625$.

[20] L.N. Trefethen, Spectral Methods in Matlab. SIAM, Philadelphia (2000).

[21] M. Tucsnak and G. Weiss, Observation and Control for Operator Semigroups. Birkhäuser, Basel (2009). 